\title{
Novel Deep Glass Etched Microring Resonators Based on Silica-on-Silicon Technology
}

\author{
Haiyan Ou, Karsten Rottwitt, and Hugh Philipp* \\ COM·DTU, Technical University of Denmark,2800,Kgs. Lyngby, Denmark \\ ho@com.dtu.dk \\ * Now with Cornell University, LASSP, Clark Hall, Ithaca, NY 14850
}

\begin{abstract}
Microring resonators fabricated in silica-on-silicon technology using deep glass etching are demonstrated. The fabrication procedures are introduced and the transmission spectrum of a resonator is presented. @ 2005 Optical Society of America

OCIS codes: (130.3120) Integrated optics devices; (220.4000) Microstructure fabrication
\end{abstract}

\section{Introduction}

Microring resonators have attracted intense research as fabrication techniques have allowed highquality waveguides to be produced on high index-contrast platforms. Since ring resonators support travelling wave resonant modes, a single ring may be used to completely extract a particular wavelength from a signal bus [1]. Microring resonators are ideal candidates for very large-scale integrated (VLSI) photonic circuits, since they provide a wide range of optical signal processing functions while being ultracompact [2].

Microring resonators have been fabricated by using high index-contrast $\mathrm{Si} / \mathrm{SiO}_{2}$ [3], $\mathrm{Ta}_{2} \mathrm{O}_{5} / \mathrm{SiO}_{2}$ [4], GaAs/AlGaAs [5], InGaAsP/InP [6], polymer [7,8], and $\mathrm{Si}_{3} \mathrm{~N}_{4} / \mathrm{SiO}_{2}[9,10]$. The high modal confinement of high index contrast waveguides allows small-radii, low-loss bends to be made. Low loss around tight bends makes large free spectral range resonators with high quality factors and finesse possible. Since silica-on-silicon technology has an index contrast on the order of 0.01, low-loss bends with radii smaller than a few millimeters are generally not possible [11]. However, with the development of the deep glass etching technology [12], it will be shown that microring resonators combining the silica-on-silicon technology with deep trenches etching are possible.

In this paper, we present the first experimental result of what we believe is the first implementation of a microring resonator in silica-on-silicon technology using deep glass etching. The additional functionality offered by the deep etched resonators could be significant, since it adds a compact nonreflective resonator to a well-developed, commercially viable integrated optics platform.

\section{Fabrication of microring resonators}

The fabrication of the silica-on-silicon microring resonators consists of two main steps. The first step is the deposition of three waveguide layers: the buffer, the core and the top-cladding. The processing starts with a 4 inch silicon wafer. The buffer layer is a $6 \mu \mathrm{m}$ thick layer of pure silicon dioxide, grown by the thermal oxidization of the silicon substrate. The core is a $3.5 \mu \mathrm{m}$ thick Ge-doped glass $\left(\mathrm{SiO}_{2}\right)$ layer deposited by plasma enhanced chemical vapour deposition (PECVD). The refractive index of the core $\left(n_{c}\right)$ is 1.465 at the wavelength of $1550 \mathrm{~nm}$. Since the refractive index of pure glass $\left(n_{b}\right)$ is 1.445 at $1550 \mathrm{~nm}$, the index contrast $\left(\mathrm{n}_{\mathrm{c}}-\mathrm{n}_{\mathrm{b}}\right)$ between the core and buffer is 0.02 . The top-cladding layer is Boron and Phosphorus co-doped glass (BPSG) deposited also by PECVD. The refractive index and thickness of the top-cladding, 1.445 and $6 \mu \mathrm{m}$ respectively, are matched to that of the buffer.

The second major fabrication step is deep glass etching. The deep glass etching goes through three layers of the waveguide, and the total etching depth is $15.5 \mu \mathrm{m}$. Burned resist was used as the mask material of deep glass etching in order to improve the sidewall roughness, which is a standard waveguide fabrication process used in the cleanroom at Danchip (DTU). As a result, the sidewall of the waveguide is angled $\left(82^{\circ}\right)$ instead of vertical. Two scanning electron microscope (SEM) pictures of the microring resonators after processing are shown in Fig. 1 and Fig.2.

Fig. 1 shows a top-view SEM picture of single ring resonator with the radius of $25 \mu \mathrm{m}$. The ring and the straight waveguide are designed to be connected at the coupling area. This "fused" section of waveguide can greatly increase coupling between guided modes in the bus waveguide and ring, and also introduce scattering losses in the junction [3].

Fig. 2 shows a tilted cross-sectional SEM picture of a waveguide. As seen from Fig.2, in the horizontal direction, the index contrast of the waveguide is increased from 1.465:1.445 to 1.465:1 after the trench etching. This contrast is comparable to the other high index contrast materials like $\mathrm{Si}_{3} \mathrm{~N}_{4}$. The relative refractive index difference [13], $\Delta=\left(\mathrm{n}_{\mathrm{c}}{ }^{2}-\mathrm{n}_{\mathrm{b}}{ }^{2}\right) /\left(2 \mathrm{n}_{\mathrm{c}}{ }^{2}\right)$, for Silicon rich Silicon Nitride 
(SRSN) with a silica cladding is 0.254 , while it is 0.267 for the glass-air interface. The tight confinement of the horizontal optical mode enables low-loss small radii bends in the plane of the wafer.

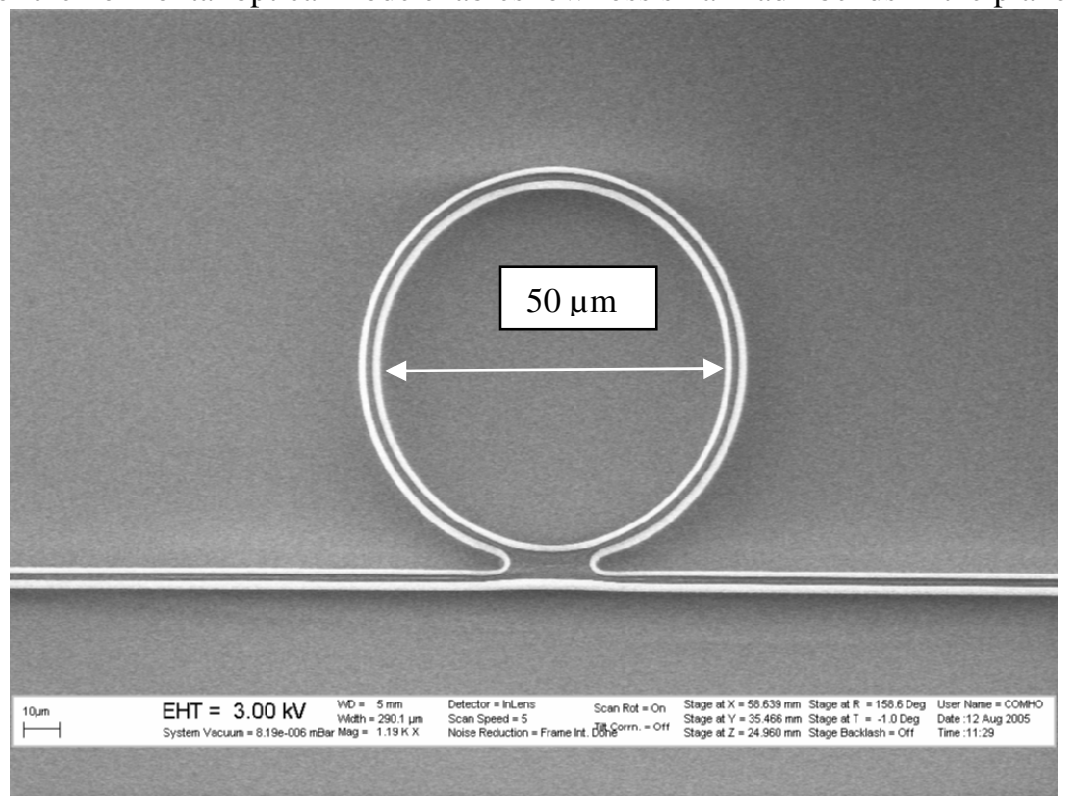

Fig. 1 A single ring and a bus waveguide picture taken by SEM. The ring shown has radius of $25 \mu \mathrm{m}$. In this picture, it is clearly shown that the bus waveguide and ring resonator are fused together in the coupling region.

Fig.2 A tilted cross-sectional SEM picture of one waveguide after the deep etching. The total etching depth is $15.5 \mu$ m, including three layers of the waveguide: top-cladding, core and buffer layer. A trapezoidal core layer is highlighted. The widths for the top and bottom of the core layer are $3.4 \mu \mathrm{m}$ and $3.9 \mu \mathrm{m}$ respectively. The thickness of the core layer is $3.5 \mu \mathrm{m}$.

\section{Measurement of the ring resonators}

The transmission of a straight waveguide coupled to one ring resonator was measured using an ANDO optical spectrum analyser (OSA) synchronised with a tunable laser source (TLS). Polarization was adjusted by a polarization controller to maximise the depth of the transmission dips corresponding to the resonant mode of the ring.

\section{Results and discussions}

A transmission spectrum of the one ring resonator was shown in Fig.3.

From the spectrum, FSR is read to be $2.64 \mathrm{~nm}$. A relationship between the radius of the ring R, the effective group index $n_{g}$, and the FSR is given by the formula: $F S R=\frac{\lambda^{2}}{2 \pi R n_{g}}$, where $\lambda$ is

wavelength. Using the measured value of $F S R=2.64 \mathrm{~nm}$, and a wavelength of $\lambda=1550 \mathrm{~nm}$ in the 
formula, the effective group index of the resonant mode was found to be $n_{g}=1.45$. This value is in good agreement with the structure of the waveguide we used.

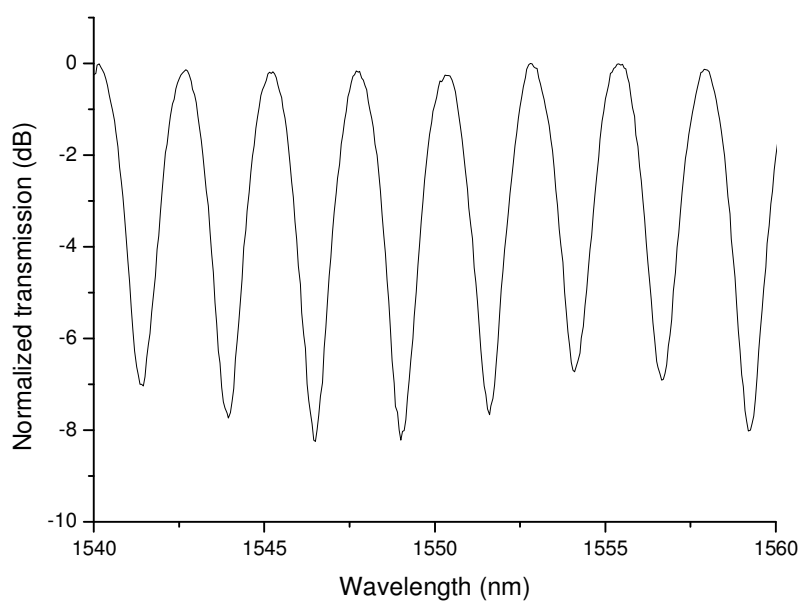

Fig.3 Transmission spectrum of one ring resonator with radius of $100 \mu \mathrm{m}$

Ring resonators typically have a Lorentzian spectral response at the resonant wavelengths. Figure 3 shows a spectrum that has a low finesse periodic response. This may be caused by sidewall roughness [14] and other sources of loss in the resonator (e.g. losses induced by the multi-mode coupling region).

Better performance is expected with improved processing and waveguide design. For example, using deep glass etched near-vertical side-walls, that have been demonstrated [12], will make tighter tolerances in the coupling region possible. Furthermore, sidewall roughness can be reduced dramatically by careful processing optimization. We believe that this technology will be significantly improved with more research effort.

\section{Conclusion}

We present for the first time to the best of our knowledge, microring resonators fabricated based on silica-on-silicon planar lightwave circuit technology and deep glass etching technology. The resonant spectrum was shown and the group index of the waveguide was extracted from the free spectral range. This value is consistent with the magnitude expected. This novel deep etched microring resonator adds significant functionality to a mature silica-on-silicon waveguide platform.

\section{References}

[1] Little, B. E., Chu, S. T., and Haus, H.A., et al, “Microring resonator channel dropping filters", J. Lightwave Technol, 15, 998-1005 (1997) .

[2] Little, B. E., Chu, S. T., and Pan, W., et al., “Microring resonator arrays for VLSI Photonics”, IEEE Photon. Technol. Lett., 12, 323-325 (2000).

[3] Little, B. E., Foresi, J. S., and Steinmeyer, G., et al, "Ultra-compact Si-SiO2 microring resonator optical channel dropping filters', IEEE Photon. Technol. Lett., 10, 549-551 (1998).

[4] Little, B. E., Chu, S. T., and Pan, W., et al, "Vertically coupled glass microring resonator channel dropping filters", IEEE Photon. Technol. Lett., 11, 215-217 (1999).

[5] Van, V., Ibrahim, T.A., and Ritter, K., et al, "All-optical nonlinear switching in GaAs-AlGaAs microring resonators", IEEE Photon. Technol. Lett., 14, 74-76 (2002).

[6] Grover, R., Absil, P. P, and Van, V., et al, "Vertically coupled GaInAsP-InP microring resonators", Opt. Lett., 26, 506-508 (2001).

[7] Lee, H.-P, Park, J.-J, and Ryoo, H.-H., et al., “'Resonance characteristics of waveguide-coupled polyimide microring resonator', Opt. Mat, 21, 535-541 (2002).

[8] Rabiei, P., Steier, W. H., Zhang, C., and Dalton, L. R., “Polymer micro-ring filters and modulators", J. Lightwave Technol., 20, 1968-1975 (2002).

[9] Sparacin, D. K., Hong, C. Y., and Kinerling, L. C., et al., "Trimming of microring resonators by photo-oxidation of a plasmapolymerized organosilane cladding material', Opt. Lett., 30, 2251-2253 (2005).

[10] Philipp, H. T., Andersen, K. N., Svendsen, W., and Ou, H., “Amorphous silicon rich silicon nitride optical waveguides for high density integrated optics", Electron. Lett., 40, 419-421 (2004).

[11]. Ou, H., "Different index contrast silica-on-silicon waveguides by PECVD', Electron. Lett., 39, $212-213$ (2003).

[12] Ou, H., "Trenches for building blocks of advanced planar components", IEEE Photon. Lett., 16, 1334-1336, (2004).

[13] Okamoto K., Fundamentals of optical waveguides, Academic Press, (2000).

[14] Little, B. E., Laine, J. -P, and Chu, S. T., "Surface-roughness-induced contradirectional coupling in ring and disk resonators', Opt. Lett., 22, 4-6, (1997). 